\title{
Existence of Equilibria with a Tight Marginal Pricing Rule
}

\author{
J.-M. Bonnisseau*and B. Cornet ${ }^{\dagger}$
}

December 1, 2009

\begin{abstract}
This paper deals with the existence of marginal pricing equilibria when it is defined by using a new and tighter normal cone introduced by B. Cornet and M.O. Czarnecki. The main interest of this new definition of the marginal pricing rule comes from the fact that it is more precise in the sense that the set of prices satisfying the condition is smaller than the one given by the Clarke's normal cone. The counterpart is that it is not convex valued, which leads to some mathematical difficulties in the existence proof. The result is obtained through an approximation argument under the same assumptions as in the previous existence results.
\end{abstract}

Keywords: General economic equilibrium, increasing returns, marginal pricing rule, existence

JEL Classification Numbers: C62, D50, D51

\section{Introduction}

Guesnerie (1975) is the first who studied the second welfare theorem in a general equilibrium setting with non-convex production sets at the level of

\footnotetext{
${ }^{*}$ Centre d'Economie de la Sorbonne,Université Paris 1 Panthéon-Sorbonne, 106 - 112 Boulevard de l'Hôpital, 75647 Paris Cedex 13, France, email:Jean-Marc.Bonnisseau@univ-paris1.fr

†University of Kansas and Université Paris 1, e-mail: cornet@ku.edu, cornet@univ-paris1.fr
} 
generality of Debreu (1959). To modelize the marginal cost pricing rule, he considered the normal cone of Dubovickii and Miljutin, that is, the firm follows the marginal cost pricing rule at a production $y$ for a price vector $p$ if $p$ belongs to the normal cone of Dubovickii and Miljutin of the production set at $y$. This definition allows to cover several cases: when the production set is convex, then we recover the standard profit maximizing behavior, when the production set is smooth, the unique normalized price satisfying the marginal cost pricing rule is the unique normalized outward normal vector, and, when the production set is defined by a finite set of smooth inequality constraints satisfying a qualification condition, the normal cone is generated by the gradient vectors of the binding constraints.

Later, Cornet (1990) (but the first version was written in 1982) proposes to use the Clarke's normal cone (see Clarke (1983)) to represent the marginal pricing rule for the existence problem. Indeed, this cone exhibits three fundamental properties: when the production set is closed and satisfies the free-disposal assumption, the Clarke's normal cone is convex, has a closed graph, and is not reduced to $\{0\}$ for a weakly efficient production. These properties were used in Bonnisseau-Cornet (1990) to prove the existence of marginal pricing equilibria with several producers.

Then, Khan (1999) (but the first version was written in the eighties) considers the limiting normal cone to extend the second welfare theorem. This cone is not necessarily convex and may be strictly smaller than the Clarke's normal cone. After him, several extensions were made in infinite dimensional spaces. Nevertheless the example of Beato and Mas-Colell (1983) shows that an equilibrium may not exists with the proximal normal cone or with the cone of Dubovickii and Miljutin, although an equilibrium exists with the Clarke's nomal cone.

The major drawback of the Clarke's normal cone is that it is too large in the sense that it is defined as the convex hull of the limiting normal cone. So, some vectors belong to the Clarke's normal cone since they are a convex combination of normal vectors but they do not satisfy a "normality" condition. Note also that Jouini (1988) exhibits a production set where the Clarke's normal cone is the positive orthant for every weakly efficient production. So, in that case, the marginal pricing rule puts no restriction on the firm's behavior.

In Bonnisseau et al (2005), a tighter definition of the marginal pricing rule is derived from a new notion of normal cone, called intermediate normal cone, between the limiting and the Clarke's normal cones introduced in Cornet- 
Czarnecki (2001). An example given in this paper, shows that it may be strictly smaller when the production set exhibits some kind of inward kinks. As for the previous definitions of the marginal pricing rules, the new definition coincides with the profit maximizing rule when the production set is convex and with the outward normal vector when the production set is smooth.

The problem raised by this new definition is that it does not lead to a convex valued pricing rule. In the previous proofs of the existence of equilibria in non-convex economies, the convexity of the set of prices satisfying the marginal pricing rule at a given production is crucial to apply a fixed-point theorem. Furthermore, the link between the marginal pricing rule and the geometry of the production set through the sub-differential of a transformation function is at the heart of the argument. We loose both with the new approach.

The purpose of this article is to provide an existence proof of a marginal pricing equilibrium with this tighter notion under the same assumptions as in the previous results with the Clarke's normal cone (see Bonnisseau (1992), Bonnisseau-Cornet (1990a,1991), Bonnisseau-Jamin (2004)). We overcome the difficulties mentioned above by considering an approximation argument and a limit argument. It is based on a result by Cornet and Czarnecki (2001) about approximation of compact epi-lipschitzian sets. But we also adapt the usual argument, which is based on a Morse's Lemma for non-differentiable mapping (Bonnisseau-Cornet (1990b)).

\section{The model and the existence result}

We consider an economy with $\ell$ commodities, $m$ consumers and $n$ producers. The commodity space is $\mathbb{R}^{\ell}$ and the commodities are indexed by $h=1, \ldots, \ell$. The consumers are indexed by $i=1, \ldots, m$. The consumption set $X_{i} \subset \mathbb{R}^{\ell}$ is the subset of all possible consumptions for consumer $i$, given her physical constraints. The tastes of this consumer are described by a binary preference relation $\preceq_{i}$ on $X_{i}$. The firms are indexed by $j=1, \ldots, n$. The technological possibilities of firms $j$ are represented by its production set $Y_{j} \subset \mathbb{R}^{\ell}$. Finally, $\omega$ denotes the initial endowments of the economy.

The wealth of the $i$ th consumer is given by a function $r_{i}: \mathbb{R}^{\ell} \backslash\{0\} \times$ $\prod_{j=1}^{n} \partial Y_{j}$ to $\mathbb{R}$, i.e., given the price vector $p \in \mathbb{R}^{\ell} \backslash\{0\}$ and the productions $\left(y_{j}\right) \in \prod_{j=1}^{n} \partial Y_{j}$, the wealth of consumer $i$ is $r_{i}\left(p,\left(y_{j}\right)\right)$. This abstract wealth structure clearly encompasses the case of a private ownership econ- 
omy, in which $r_{i}\left(p,\left(y_{j}\right)\right)=\sum_{j=1}^{n} \theta_{i j} p \cdot y_{j}+p \cdot \omega_{i}$, where the $\theta_{i j}$ denote the consumers' shares in the production processes and satisfy $\theta_{i j} \geq 0$ for all $i, j$ and $\sum_{i=1}^{m} \theta_{i j}=1$ for all $j$, and where the $\omega_{i}$ denote the consumers' initial individual endowments and satisfy $\sum_{i=1}^{m} \omega_{i}=\omega$.

We posit the following assumptions on the fundamentals of the economy, which are maintained throughout the paper. 1 denotes the vector of $\mathbb{R}^{\ell}$, with all coordinates equal to 1 and $X=\sum_{i=1}^{m} X_{i}+\mathbb{R}_{+}^{\ell}-\{\omega\}$.

Assumption (C) For every $i, X_{i}$ is a non-empty, closed, convex bounded below subset of $\mathbb{R}^{\ell}, \preceq_{i}$ is a continuous, convex and non-satiated complete preorder $^{1}$ on $X_{i}$, and $r_{i}$ is a continuous function on $\mathbb{R}^{\ell} \backslash\{0\} \times \prod_{j=1}^{n} \partial Y_{j}$, satisfying $r_{i}\left(\alpha p,\left(y_{j}\right)\right)=\alpha r_{i}\left(p,\left(y_{j}\right)\right)$ and $\sum_{i=1}^{m} r_{i}\left(p,\left(y_{j}\right)\right)=p \cdot\left(\omega+\sum_{j=1}^{n} y_{j}\right)$ for every $\alpha>0$ and every $\left(p,\left(y_{j}\right)\right) \in \mathbb{R}^{\ell} \backslash\{0\} \times \prod_{j=1}^{n} \partial Y_{j}$.

Assumption (P) For every $j, Y_{j}$ is a nonempty, closed subset of $\mathbb{R}^{\ell}$, different from $\mathbb{R}^{\ell}$ and $Y_{j}-\mathbb{R}_{+}^{\ell}=Y_{j}$.

Assumption (B) For every $t \geq 0, A_{t}=\left\{\left(y_{j}\right) \in \prod_{j=1}^{n} \partial Y_{j} \mid \sum_{j=1}^{n} y_{j}+t \mathbf{1} \in\right.$ $X\}$ is bounded.

The first assumption is the standard assumption on the preferences and the revenue functions. In Assumption $(\mathrm{P})$, the production sets are not assumed to be convex but only to satisfy the free-disposal assumption, which is compatible with fixed cost, increasing returns and other types of nonconvexities. Assumption B means that the feasible productions are bounded even if one increases the initial endowments.

We now introduce the definition of the marginal pricing rule, which differs from the standard one. Indeed, instead of considering the Clarke's normal cone to the production set as in Cornet (1990) or Bonnisseau-Cornet (1990), we consider the intermediate normal cone introduced by Cornet-Czarnecki (2001). It is always contained in the Clarke's normal cone but it is not always convex valued. We refer to Bonnisseau et al. (2005) for an example of a production set satisfying Assumption $\mathrm{P}$ and such that the intermediate normal cone is non-convex. and strictly smaller than the Clarke's normal cone at the origin.

\footnotetext{
${ }^{1} \preceq_{i}$ is a complete, reflexive, transitive binary relation, and, for every $x_{i} \in X_{i}$, the subsets $\left\{x \in X_{i} \mid x \preceq_{i} x_{i}\right\}$ and $\left\{x \in X_{i} \mid x_{i} \preceq_{i} x\right\}$ are closed, the subset $\left\{x \in X_{i} \mid x_{i} \preceq_{i} x\right\}$ is convex, and, there exists $x \in X_{i}$ such that $x_{i} \prec_{i} x$.
} 
To introduce the new notion of normal cone, we consider the distance function $d_{Y_{j}}$ to $Y_{j}$ associated to the usual Euclidean norm in $\mathbb{R}^{\ell}$ and its generalized gradient. We know that $d_{Y_{j}}$ is Lipschitz and, thus, from Rademacher's Theorem, almost everywhere differentiable. We denote by $\operatorname{dom}\left(\nabla d_{Y_{j}}\right)$ the domain on which $d_{Y_{j}}$ is differentiable. The Clarke's generalized gradient $\partial d_{Y_{j}}\left(y_{j}\right)$ of $d_{Y_{j}}$ at $y_{j}$ is defined as:

$$
\partial d_{Y_{j}}\left(y_{j}\right)=\mathrm{co} \limsup _{y_{j}^{\prime} \in \operatorname{dom}\left(\nabla d_{Y_{j}}\right), y_{j}^{\prime} \rightarrow y_{j}} \nabla d_{Y_{j}}\left(y_{j}^{\prime}\right)
$$

For $y_{j} \in Y_{j}$, the intermediate normal cone is defined as follows:

$$
N_{Y_{j}}^{I}\left(y_{j}\right)=\cup_{t \geq 0} t \limsup _{y_{j}^{\prime} \notin Y_{j}, y_{j}^{\prime} \rightarrow y_{j}} \partial d_{Y_{j}}\left(y_{j}^{\prime}\right)
$$

We recall the following elementary properties of the intermediate normal cone. The proof can be found in Cornet-Czarnecki (2001).

Proposition 2.1 Under Assumption P, for every $y_{j} \in Y_{j}$,

a) $N_{Y_{j}}^{I}\left(y_{j}\right) \subset \mathbb{R}_{+}^{\ell}$ and $\{0\} \neq N_{Y_{j}}^{I}\left(y_{j}\right)$ if $y_{j} \in \partial Y_{j}$;

b) If $Y_{j}$ is convex, $N_{Y_{j}}^{I}\left(y_{j}\right)=\left\{p \in \mathbb{R}^{\ell} \mid p \cdot y_{j} \geq p \cdot y_{j}^{\prime}, \forall y_{j}^{\prime} \in Y_{j}\right\}$.

We can now define the marginal pricing rule.

Definition 2.1 A producer follows the marginal pricing rule at the production $y_{j} \in \partial Y_{j}$ for the price $p \in \mathbb{R}^{\ell} \backslash\{0\}$ if $p \in N_{Y_{j}}^{I}\left(y_{j}\right)$.

In the following, the price are normalized in the simplex $S$ of $\mathbb{R}^{\ell}$,

$$
S=\left\{p \in \mathbb{R}_{+}^{\ell} \mid \sum_{h=1}^{\ell} p_{h}=1\right\}
$$

Indeed, the free-disposal assumption implies that the equilibrium prices are nonnegative and the other assumptions implies that the equilibrium prices can be normalized in the simplex without any loss of generality. We will consider the marginal pricing rule as a correspondence $M P_{j}$ from $\partial Y_{j}$ to $S$ defined by $M P_{j}\left(y_{j}\right)=N_{Y_{j}}^{I}\left(y_{j}\right) \cap S$.

We now define formally a marginal pricing equilibrium of the economy. 
Definition 2.2 An element $\left(\left(x_{i}^{*}\right),\left(y_{j}^{*}\right), p^{*}\right)$ in $\left(\mathbb{R}^{\ell}\right)^{m} \times\left(\mathbb{R}^{\ell}\right)^{n} \times S$ is a marginal pricing equilibrium of the economy $\mathcal{E}=\left(\left(X_{i}, \preceq_{i}, r_{i}\right)_{i=1}^{m},\left(Y_{j}\right)_{j=1}^{n}, \omega\right)$ if:

(a) for every $i, x_{i}^{*}$ is a greater element for $\preceq_{i}$ in $B_{i}\left(p^{*},\left(y_{j}^{*}\right)\right)=\left\{x_{i} \in X_{i} \mid\right.$ $\left.p^{*} \cdot x_{i} \leq r_{i}\left(p^{*},\left(y_{j}^{*}\right)\right)\right\}$

(b) for every $j, y_{j}^{*} \in \partial Y_{j}$ and $p^{*} \in M P_{j}\left(y_{j}^{*}\right)$;

(c) $\sum_{i=1}^{m} x_{i}^{*}=\sum_{j=1}^{n} y_{j}^{*}+\omega$.

If we consider only the production sector, we define the set of production equilibria as

$$
P E=\left\{\left(p,\left(y_{j}\right)\right) \in S \times \prod_{j=1}^{n} \partial Y_{j} \mid p \in M P_{j}\left(y_{j}\right) \forall j=1, \ldots, n\right\}
$$

We are now able to state the existence result. In the following, $d_{X}^{\infty}$ denotes the distance function to the set $X$ associated to the sup-norm.

Theorem 2.1 An economy $\mathcal{E}$ satisfying Assumptions $(C),(P)$ and $(B)$ has a marginal pricing equilibrium if

Assumption (S) for every $\left(p,\left(y_{j}\right)\right) \in P E$,

$$
p \cdot \sum_{j=1}^{n} y_{j}>\inf p \cdot X-d_{X}^{\infty}\left(\sum_{j=1}^{n} y_{j}\right)
$$

Assumption (R) for every $\left(p,\left(y_{j}\right)\right) \in P E$, if $\left(y_{j}\right) \in A_{0}$, then $r_{i}\left(p,\left(y_{j}\right)\right)>$ $\inf p \cdot X_{i}$, for all $i=1, \ldots, m$.

The assumptions are actually identical to those of Bonnisseau-Cornet (1990) but the marginal pricing rule has not the same definition. Since the intermediate normal cone is smaller than the Clarke's one, this result is more precise. Note that an important difference comes from the fact that the marginal pricing rule is not convex valued whereas all previous existence results assume that the pricing rules are convex valued.

Note also that our formulation of the survival assumption is not the same. But it is equivalent since the fact that $X+\mathbb{R}_{+}^{\ell}=X$, implies that for all $x \in \mathbb{R}^{\ell}, x+d_{X}^{\infty}(x) \mathbf{1} \in \partial X$ and $x+d_{X}^{\infty}(x) \mathbf{1}$ is one of the closest point to $x$ in $X$. From this remark, since $p \in S$, for all $\left(p,\left(y_{j}\right)\right) \in P E$, one has $p \cdot \sum_{j=1}^{n} y_{j} \geq \inf p \cdot X-d_{X}^{\infty}\left(\sum_{j=1}^{n} y_{j}\right)$. So, Assumption (S) requires that the inequality is always strict. 


\section{Proof of the existence result}

The proof is divided in three steps: first, we truncate the production sets and we approximate the truncated production sets by using the following result of Cornet-Czarnecki (2001). $N_{Y}^{C}$ denotes the Clarke's normal cone.

Theorem 3.1 Let $Z$ be a compact epi-Lipschitizian subset of $\mathbb{R}^{\ell}$. $Z$ admits a smooth normal approximation $\left(Z_{k}\right)_{k \in \mathbb{N}}$ in the sense that:

(i) for every $k, Z_{k}$ is a compact and smooth subset of $\mathbb{R}^{\ell}$, that is a closed $C^{\infty}$ submanifold with boundary of $\mathbb{R}^{\ell}$ of full dimension;

(ii) for every $k, Z_{k+1} \subset Z_{k} \subset\left\{z \in \mathbb{R}^{\ell} \mid d_{Z}(z)<1\right\}$ and $Z=\cap_{k \in \mathbb{N}} Z_{k}$;

(iii) $\lim \sup _{k \rightarrow \infty} G\left(N_{Z_{k}}^{C}\right) \subset G\left(N_{Z}^{I}\right)$.

Then, using the normal cone to the smooth approximations, we define continuous functions, which approximates the marginal pricing rules. We also use a Morse's Lemma to modify these functions in such a way that the bounded losses assumption be satisfied by the approximate pricing rule. In the third step, we use an existence result (Bonnisseau-Jamin (2004)) to get an approximate equilibrium, and, we end the proof by a limit argument. The proof of the lemmas are given in Appendix.

\subsection{Approximation of the production sets}

We first recall some properties of the production sets, which come from the free disposal assumption. The following Lemma gathers the results of Lemma 5.1 in Bonnisseau-Cornet (1988) and Lemma 4.2 in BonnisseauCornet (1990), with a slight generalization from Bonnisseau-Jamin (2004). Let $H$ be the hyperplane defined by $H=\left\{x \in \mathbb{R}^{\ell} \mid x \cdot \mathbf{1}=1\right\}$ and let $C$ be a pointed closed convex cone such that $\mathbf{1} \in \operatorname{int} C$. We first state a variant of Assumption (P) in which $\mathbb{R}_{+}^{\ell}$ is replaced by $C$.

Assumption $\left(\mathbf{P}_{C}\right)$ For every $j, Y_{j}$ is a nonempty closed subset of $\mathbb{R}^{\ell}$ different from $\mathbb{R}^{\ell}$ and $Y_{j}-C=Y_{j}$.

Lemma 3.1 Let us assume that Assumption $\left(P_{C}\right)$ holds true. Then, for every $j$, for every $s \in \mathbf{1}^{\perp}$, there is a unique real number, denoted by $\lambda_{j}(s)$, such that $s-\lambda_{j}(s) \mathbf{1} \in \partial Y_{j}$. The function $\lambda_{j}: \mathbf{1}^{\perp} \rightarrow \mathbb{R}$ is Lipschitz continuous, 
and the mapping $\Lambda_{j}: s \in \mathbf{1}^{\perp} \mapsto s-\lambda_{j}(s) \mathbf{1} \in \partial Y_{j}$ is an homeomorphism. The inverse of $\Lambda_{j}$ is the restriction of the orthogonal projection on $\mathbf{1}^{\perp}$ to $\partial Y_{j}$.

$$
Y_{j}=\left\{y_{j} \in \mathbb{R}^{\ell} \mid \lambda_{j}\left(\operatorname{proj}_{\mathbf{1}^{\perp}}\left(y_{j}\right)\right)+\left(y_{j} \cdot \mathbf{1} / \ell\right) \leq 0\right\} .
$$

The generalized gradient of $\lambda_{j}$ at $s \in \mathbf{1}^{\perp}$ is given by:

$$
\partial \lambda_{j}\left(s_{j}\right)=\left(N_{Y_{j}}\left(\Lambda_{j}\left(s_{j}\right)\right) \cap H\right)-\{(1 / \ell) \mathbf{1}\}
$$

and the correspondence $\partial \lambda_{j}$, from $\mathbf{1}^{\perp}$ into itself, is upper hemi-continuous with non-empty, convex, compact values.

Note that $Y_{0}=-X$ satisfies also Assumption $\left(\mathrm{P}_{C}\right)$ from Assumptions (C). We denote by $\lambda_{0}$ et $\Lambda_{0}$ the mappings associated to $Y_{0}$. We also define the mapping $\Lambda$ from $\left(\mathbf{1}^{\perp}\right)^{n}$ to $\prod_{j=1}^{n} \partial Y_{j}$ by $\Lambda(s)=\left(\Lambda_{j}\left(s_{j}\right)\right)$.

Let $\theta$ be the function defined on $\left(\mathbf{1}^{\perp}\right)^{n}$ by:

$$
\theta(s)=\sum_{j=1}^{n} \lambda_{j}\left(s_{j}\right)+\lambda_{0}\left(-\sum_{j=1}^{n} s_{j}\right) .
$$

Since $X$ satisfies $X+\mathbb{R}_{+}^{\ell}=X$, we remark that $\theta\left(\left(\operatorname{proj}_{\mathbf{1}^{\perp}}\left(y_{j}\right)\right)\right)=d_{X}^{\infty}\left(\sum_{j=1}^{n} y_{j}\right)$ if $\sum_{j=1}^{n} y_{j} \notin X$. Indeed, $\sum_{j=1}^{n} y_{j}+\theta\left(s_{j}\right) \mathbf{1}=\sum_{j=1}^{n} s_{j}+\lambda_{0}\left(-\sum_{j=1}^{n} s_{j}\right) \mathbf{1}=$ $-y_{0} \in \partial X$, where $s_{j}=\operatorname{proj}_{1^{\perp}}\left(y_{j}\right)$. Consequently, the open ball for the supnorm of center $\sum_{j=1}^{n} y_{j}$ and radius $\theta\left(\left(s_{j}\right)\right)$ is included in $-y-\mathbb{R}_{++}^{\ell}$, which does not intersect $X$.

Note that $\theta$ is Lipschitz continuous, and, for every real number $t \geq 0$, we have:

$$
M_{t}=\Lambda^{-1}\left(A_{t}\right)=\left\{s \in\left(\mathbf{1}^{\perp}\right)^{n} \mid \theta(s) \leq t\right\},
$$

and

$$
A_{t}=\left\{\left(y_{j}\right) \in \prod_{j=1}^{n} \partial Y_{j} \mid \theta\left[\left(\operatorname{proj}_{\mathbf{1}^{\perp}}\left(y_{j}\right)\right)\right] \leq t\right\} .
$$

Note that for all $t \geq 0, A_{t}$ and $M_{t}$ are bounded and closed and the correspondence $t \rightarrow M_{t}$ is uuper semi-continuous.. Let $r_{1}>0$ such that $M_{0} \subset\left[B_{1^{\perp}}\left(0, r_{1}\right)\right]^{n}$. Let $\bar{t}>\sup \left\{\theta(s) \mid s \in\left[\bar{B}_{1^{\perp}}\left(0, r_{1}\right)\right]^{n}\right\}$. Note that $\left[\bar{B}_{\mathbf{1}^{\perp}}\left(0, r_{1}\right)\right]^{n} \subset \operatorname{int} M_{\bar{t}}$. Let $r_{2}>r_{1}$ such that $M_{\bar{t}} \subset\left[B_{1^{\perp}}\left(0, r_{2}\right)\right]^{n}$. Finally, let $r_{2}^{\prime}>r_{2}$ and $\underline{\tau}>0$ such that for all $s \in\left[\bar{B}_{1^{\perp}}\left(0, r_{2}^{\prime}\right)\right]^{n}$, for all $j=1, \ldots, n$, $\Lambda_{j}\left(s_{j}\right) \gg-\underline{\tau} \mathbf{1}$.

For all $j$, let

$$
Z_{j}=\left[\Lambda_{j}\left(\bar{B}_{\mathbf{1}^{\perp}}\left(0, r_{2}^{\prime}\right)\right)-\mathbb{R}_{+}^{\ell}\right] \cap\left[\{-\bar{\tau} \mathbf{1}\}+\mathbb{R}_{+}^{\ell}\right]
$$


Lemma 3.2 For all $j, Z_{j}$ is a nonempty compact epilipschitzian subset of $\mathbb{R}^{\ell}$. For all $s_{j} \in \bar{B}_{\mathbf{1}^{\perp}}\left(0, r_{2}\right), \Lambda_{j}\left(s_{j}\right) \in \partial Z_{j}$ and $N_{Z_{j}}^{I}\left(\Lambda_{j}\left(s_{j}\right)\right)=N_{Y_{j}}^{I}\left(\Lambda_{j}\left(s_{j}\right)\right)$.

We now apply Theorem 3.1 to $Z_{j}$. There exists a sequence $\left(Z_{j}^{k}\right)_{k \in \mathbb{N}}$ satisfying:

(i) for every $k, Z_{j}^{k}$ is a compact and smooth subset of $\mathbb{R}^{\ell}$, that is a closed $C^{\infty}$ submanifold with boundary of $\mathbb{R}^{\ell}$ of full dimension;

(ii) for every $k, Z_{j}^{k+1} \subset Z_{j}^{k} \subset\left\{y \in \mathbb{R}^{\ell} \mid d_{Z_{j}}(y)<1\right\}$ and $Z_{j}=\cap_{k \in \mathbb{N}} Z_{j}^{k}$;

(iii) $\lim \sup _{k \rightarrow \infty} G\left(N_{Z_{j}^{k}}^{C}\right) \subset G\left(N_{Z_{j}}^{I}\right)$.

Let $\varepsilon_{j}^{k}=\sup \left\{d_{Z_{j}}^{\infty}\left(z_{j}\right) \mid z_{j} \in Z_{j}^{k}\right\}$. We remark that Lemma 3.2 (ii) implies that the sequence $\left(\varepsilon_{j}^{k}\right)$ converges to 0 and $Z_{j}^{k}$ is included in $Y_{j}+\left\{\varepsilon_{j}^{k} \mathbf{1}\right\}$. We denote by $\varepsilon^{k}=\sum_{j=1}^{n} \varepsilon_{j}^{k}$.

Let $\tau>\underline{\tau}$ close enough to $\underline{\tau}$ and $\eta>0$ small enough such that for all $s \in\left[\bar{B}_{1^{\perp}}\left(0, r_{2}\right)\right]^{n}$, for all $j=1, \ldots, n$,

$$
\Lambda_{j}\left(s_{j}\right) \gg-\tau \mathbf{1}
$$

and

$$
M_{\bar{t}+\eta} \subset\left[B_{\mathbf{1}^{\perp}}\left(0, r_{2}\right)\right]^{n}
$$

We now choose a nonempty closed convex cone $C$ included in $\{0\} \cup \mathbb{R}_{++}^{\ell}$ such that $1 \in \operatorname{int} C$. Let $X^{C}=\sum_{i=1}^{m} X_{i}+C-\omega$. Note that $-X^{C}$ satisfies the Assumptions of Lemma 3.1, so we can define the mappings $\lambda_{0}^{C}$ and $\Lambda_{0}^{C}$ associated to $-X^{C}$. Note that $\lambda_{0}^{C} \geq \lambda_{0}$. We let:

$$
\theta^{C}(s)=\sum_{j=1}^{n} \lambda_{j}\left(s_{j}\right)+\lambda_{0}^{C}\left(-\sum_{j=1}^{n} s_{j}\right)
$$

For further approximations, we choose $C$ large enough according to the next lemma where $\tau$ and $\eta$ are defined in .(1) and (2)

Lemma 3.3 There exists a nonempty closed convex cone $C$ included in $\{0\} \cup$ $\mathbb{R}_{++}^{\ell}$ such that $\mathbf{1} \in \operatorname{int} C$ and, for all $s \in \bar{B}_{\mathbf{1}^{\perp}}\left(0, n r_{2}\right), \lambda_{0}^{C}(s)-\lambda_{0}(s)<\eta$.

Note that the negative polar cone of $C, C^{\circ}$, satisfies $-\mathbb{R}_{+}^{\ell} \backslash\{0\} \subset \operatorname{int} C^{\circ}$. We let $Y_{j}^{k}=Z_{j}^{k}-C$. 
Lemma 3.4 There exists an integer $\underline{k}$ such that for all $k \geq \underline{k}$,

(i) for all $z_{j} \in \partial Z_{j}^{k} \cap\left(\{-\tau \mathbf{1}\}+\mathbb{R}_{+}^{\ell}\right), N_{Z_{j}^{k}}^{C}\left(z_{j}\right) \backslash\{0\} \subset-\operatorname{int} C^{\circ}$;

(ii) Assumptions $\left(P_{C}\right)$ and $(B)$ are satisfied by $\left(Y_{j}^{k}\right)$;

(iii) For all $j, Y_{j}^{k} \subset Y_{j}+\left\{\varepsilon_{j}^{k} \mathbf{1}\right\}$ and $Y_{j}^{k} \cap\left[\{-\tau \mathbf{1}\}+\mathbb{R}_{++}^{\ell}\right]=Z_{j}^{k}$.

Using Lemma 3.1, for all $k \geq \underline{k}$, we can define the mappings $\lambda_{j}^{k}, \Lambda_{j}^{k}$ associated to $Y_{j}^{k}$. Then, we define the mapping $\theta^{k}$ and the sets $M_{t}^{k}$ as follows:

$$
\begin{aligned}
\theta^{k}(s) & =\sum_{j=1}^{n} \lambda_{j}^{k}\left(s_{j}\right)+\lambda_{0}^{C}\left(-\sum_{j=1}^{n} s_{j}\right) . \\
M_{t}^{k} & =\left\{s \in\left(\mathbf{1}^{\perp}\right)^{n} \mid \theta^{k}(s) \leq t\right\},
\end{aligned}
$$

The following lemma summarizes the link between this mappings and sets and the original ones.

Lemma 3.5 There exists an integer $\tilde{k} \geq \underline{k}$ such that for all $k \geq \tilde{k}$,

(i) for all $j$, for all $s \in \mathbf{1}^{\perp}, \lambda_{j}(s)-\varepsilon_{j}^{k} \leq \lambda_{j}^{k}(s)$ and for all $s \in \bar{B}_{\mathbf{1}^{\perp}}\left(0, r_{2}\right)$, $\lambda_{j}^{k}(s) \leq \lambda_{j}(s)$

(ii) $M_{0}^{k} \subset M_{\varepsilon^{k}} \subset\left(B_{1^{\perp}}\left(0, r_{1}\right)\right)^{n}$;

(iii) $\sup \left\{\theta^{k}(s) \mid s \in\left(\bar{B}_{\mathbf{1}^{\perp}}\left(0, r_{1}\right)\right)^{n}\right\}<\bar{t}+\eta$;

(iv) $M_{\bar{t}+\eta}^{k} \subset M_{\bar{t}+\eta+\varepsilon^{k}} \subset\left(B_{\mathbf{1}^{\perp}}\left(0, r_{2}\right)\right)^{n}$;

\subsection{Approximation of the marginal pricing rules}

For all $j$, for all $s_{j} \in \bar{B}_{\mathbf{1}^{\perp}}\left(0, r_{2}\right)$, for all $k \geq \tilde{k}$, Lemma 3.4 (iii) implies that $\Lambda_{j}^{k}\left(s_{j}\right) \gg-\tau \mathbf{1}$ and $\Lambda_{j}^{k}\left(s_{j}\right) \in \partial Z_{j}^{k}$. From Lemma 3.4 (i) and (iii), $N_{Y_{j}^{k}}^{C}\left(\Lambda_{j}^{k}\left(s_{j}\right)\right)=N_{Z_{j}^{k}}^{C}\left(\Lambda_{j}^{k}\left(s_{j}\right)\right)$, and, this normal cone is an half line included in $-C^{\circ}$, since $Z_{j}^{k}$ is smooth. We denote by $g_{j}^{k}\left(s_{j}\right)$ the unique element of $N_{Y_{j}^{k}}^{C}\left(\Lambda_{j}^{k}\left(s_{j}\right)\right)$ in the hyperplane $H$. Note that $g_{j}^{k}$ is a continuous mapping since $Z_{j}^{k}$ is a $\mathcal{C}^{\infty}$ sub-manifold.

Lemma 3.6 There exists an integer $\hat{k} \geq \tilde{k}$ such that for all $k \geq \hat{k}$, for all $(p, s) \in H \times\left(\bar{B}_{\mathbf{1}^{\perp}}\left(0, r_{2}\right)\right)^{n}$, 
(i) if $\theta^{C}(s) \geq 0$ and $p \in-N_{X^{C}}\left(-\Lambda_{0}^{C}\left(-\sum_{j=1}^{n} s_{j}\right)\right)$, then $\left(p-g_{j}^{k}\left(s_{j}\right)\right) \neq$ $(0, \ldots, 0)$;

(ii) if $\theta^{C}(s) \leq 0$ and $p=g_{j}^{k}\left(s_{j}\right)$ for all $j$, then $r_{i}\left(p,\left(\Lambda_{j}\left(s_{j}\right)\right)\right)>\inf p \cdot X_{i}$ for all $i$.

From Clarke (1983) (Propositions 2.3.3 and 2.3.10) and Lemma 3.1, for every $s=\left(\bar{B}_{\mathbf{1}^{\perp}}\left(0, r_{2}\right)\right)^{n}, \partial \theta^{k}(s) \subset \Delta^{k}(s)$ where

$$
\Delta^{k}(s)=\left\{\left(g_{1}^{k}\left(s_{j}\right)-p, \ldots, g_{n}^{k}\left(s_{n}\right)-p\right) \mid p \in-N_{X^{C}}\left(-\Lambda_{0}^{C}\left(-\sum_{j=1}^{n} s_{j}\right)\right) \cap H\right\}
$$

and, from Clarke's normal cone properties, $\Delta$ is an upper hemi-continuous correspondence from $\left(\mathbf{1}^{\perp}\right)^{n}$ into itself with non-empty, convex, compact values.

The previous lemma implies the fundamental property of $\partial \theta^{k}$.

Lemma 3.7 For all $k \geq \hat{k}$, for all $s \in\left(\bar{B}_{\mathbf{1}^{\perp}}\left(0, r_{2}\right)\right)^{n}$, if $\theta^{k}(s) \geq 0$, then $0 \notin \Delta^{k}(s)$.

We can now use the following lemma proved in Bonnisseau-Jamin (2004) and a similar argument as the one use in this paper to build the approximate pricing rule. This lemma is actually a corollary of the Morse's Lemma proved in Bonnisseau-Cornet (1990b). $T_{\mathcal{M}}$ denotes the ordinary tangent cone of the convex analysis to $\mathcal{M}$.

Lemma 3.8 Let $E$ be a finite-dimensional Euclidean space, let $a, b \in \mathbb{R}$ such that $a<b$, let $\theta: E \rightarrow \mathbb{R}$ be a locally Lipschitz continuous function, and let $\Delta$ be a correspondence from $E$ into itself. Suppose that:

(i) the set $M_{a b}:=\{s \in E \mid a \leq \theta(s) \leq b\}$ is non-empty and compact;

(ii) $\Delta$ is an upper hemi-continuous correspondence with non-empty, convex, compact values, satisfying $\partial \theta(s) \subset \Delta(s)$ for every $s \in E$;

(iii) $0 \notin \Delta(s)$ for every $s \in M_{a b}$.

If $\mathcal{M}$ is a closed, convex, compact subset of $E$ such that $M_{a} \subset \operatorname{int} \mathcal{M} \subset M_{b}$, then there exists a continuous (homotopy) mapping $\Gamma: \partial \mathcal{M} \times[0,1] \rightarrow E$ such that, for every $s \in \partial \mathcal{M}$, 
(I) $\inf \{\Gamma(s, 0) \cdot \delta \mid \delta \in \Delta(s)\}>0$;

(II) $\Gamma(s, 1) \in-\operatorname{int} T_{\mathcal{M}}(s)$;

(III) $\Gamma(s, t) \neq 0$ for every $t \in[0,1]$.

For all $k \geq \hat{k}$, Lemmas 3.5 and 3.7 show that the Assumption of Lemma 3.8 are satisfied by the mapping $\theta^{k}, a=0, b=\bar{t}+\eta$ and $\mathcal{M}=\left(\bar{B}_{\mathbf{1}^{\perp}}\left(0, r_{1}\right)\right)^{n}$.

Thus, there exists a continuous mapping $\Gamma^{k}: \mathcal{M} \times[0,1] \rightarrow\left(\mathbf{1}^{\perp}\right)^{n}$ satisfying conditions (I), (II), and (III) of Lemma 3.8 for every $s \in \partial \mathcal{M}$.

Let $r_{1}^{\prime}>r_{1}$. We define the function $\sigma$, from $\left(\mathbf{1}^{\perp}\right)^{n}$ to $[0,1]$, by:

$$
\sigma(s)=\left\{\begin{array}{cl}
0 & \text { if } s \in \mathcal{M} \\
\frac{1}{r_{1}^{\prime}-r_{1}} \max _{j}\left\{\left\|s_{j}\right\|-r_{1}\right\} & \text { if } s \in\left(\bar{B}_{\mathbf{1}^{\perp}}\left(0, r_{1}^{\prime}\right)\right)^{n} \backslash \mathcal{M} \\
1 & \text { if } s \notin\left(\bar{B}_{\mathbf{1}^{\perp}}\left(0, r_{1}^{\prime}\right)\right)^{n}
\end{array}\right.
$$

Finally, for every $(p, s) \in H \times\left(\mathbf{1}^{\perp}\right)^{n}$,

$$
\gamma^{k}(p, s)=\left\{\begin{array}{cl}
\left(g_{j}^{k}\left(s_{j}\right)\right) & \text { if } s \in \operatorname{int} \mathcal{M} \\
\operatorname{co}\left\{\left(g_{j}^{k}\left(s_{j}\right)\right),\left((p, \ldots, p)+\Gamma^{k}(s, 0)\right)\right\} & \text { if } s \in \partial \mathcal{M} \\
\left\{(p, \ldots, p)+\Gamma^{k}\left(\operatorname{proj}_{\mathcal{M}}(s), \sigma(s)\right)\right\} & \text { if } s \notin \mathcal{M}
\end{array}\right.
$$

where $\operatorname{proj}_{\mathcal{M}}$ is the projection on $\mathcal{M}$.

We now define the pricing rule $\varphi^{k}$ from $H \times \prod_{j=1}^{n} \partial Y_{j}$ to $H^{n}$ as follows: For all $\left(p,\left(y_{j}\right)\right) \in H \times \prod_{j=1}^{n} \partial Y_{j}$, let $\left.\varphi^{k}\left(p,\left(y_{j}\right)\right)=\gamma^{k}\left(p, \operatorname{proj}_{\mathbf{1}^{\perp}}\left(y_{j}\right)\right)\right)$.

\subsection{Existence of an approximate equilibrium}

For all $k \geq \hat{k}$, we consider the economy $\mathcal{E}^{k}=\left(\left(X_{i}, \preceq_{i}, r_{i}\right)_{i=1}^{m},\left(Y_{j}\right)_{j=1}^{n}, \varphi^{k}, \omega\right)$ and we show that it satisfies the necessary conditions for the existence of a general pricing rule equilibrium given in the following theorem of BonnisseauJamin (2004). The difference between a marginal pricing equilibrium and a general pricing rule equilibrium is that we replace in Condition (b) of Definition 2.2, $p^{*} \in M P_{j}\left(y_{j}^{*}\right)$ by $\left(p^{*}, \ldots, p^{*}\right) \in \varphi^{k}\left(p^{*},\left(y_{j}^{*}\right)\right)$.

For the normalization of the prices, we consider the extended simplex $S^{C}=H \cap\left(-C^{\circ}\right)$. In the following for all $t \geq 0$, we denote by $A_{t}^{C}$ the set $\left\{\left(y_{j}\right) \in \prod_{j=1}^{n} \partial Y_{j} \mid \sum_{j=1}^{n} y_{j}+t \mathbf{1} \in X^{C}\right\}$

Theorem 3.2 The economy $\mathcal{E}^{k}$ has a general pricing rule equilibrium if it satisfies Assumption $(C),\left(P_{C}\right),(B)$, and, 
Assumption (PR) For all $\left(p,\left(y_{j}\right)\right) \in S^{C} \times \prod_{j=1}^{n} \partial Y_{j}, \varphi^{k}$ has nonempty, compact, convex values included in $H$, it is upper hemi-continuous, and, $\varphi^{k}\left(p,\left(y_{j}\right)\right) \subset\left(S^{C}\right)^{n}$ for every $\left(p,\left(y_{j}\right)\right) \in S^{C} \times A_{0}^{C}$,

Assumption ( $\left.\mathbf{R}^{\prime}\right)$ for every $\left(p,\left(y_{j}\right)\right) \in S^{C} \times \prod_{j=1}^{n} \partial Y_{j}$, if $\left(y_{j}\right) \in A_{0}^{C}$ and $(p, \ldots, p) \in \varphi^{k}\left(p,\left(y_{j}\right)\right)$, then $r_{i}\left(p,\left(y_{j}\right)\right)>\inf p \cdot X_{i}$.

Assumption (BLS) There exists a real number $t_{0} \geq 0$ such that:

(BS) for every $t \in\left[0, t_{0}\left[\right.\right.$ and every $\left(p,\left(y_{j}\right)\right) \in S^{C} \times \prod_{j=1}^{n} \partial Y_{j}$, if $\left(y_{j}\right) \in A_{t}^{C}$ and $(p, \ldots, p) \in \varphi^{k}\left(p,\left(y_{j}\right)\right)$, then $p \cdot\left(\sum_{j=1}^{n} y_{j}+t \mathbf{1}\right)>\inf p \cdot X^{C}$.

(BL) for every $t \geq t_{0}$, every $\left(p,\left(y_{j}\right)\right) \in S^{C} \times \prod_{j=1}^{n} \partial Y_{j}$, and, every $\left(q_{j}\right) \in$ $\varphi^{k}\left(p,\left(y_{j}\right)\right)$, if $p \in-N_{X^{C}}\left(\sum_{j=1}^{n} y_{j}+t \mathbf{1}\right)$, then there exists $\left(\hat{y}_{j}\right) \in A_{t_{0}}^{C}$ such that $\sum_{j=1}^{n}\left(q_{j}-p\right) \cdot\left(y_{j}-\hat{y}_{j}\right)>0$.

To simplify the exposition, we have chosen to state weaker assumptions than in Bonnisseau-Jamin (2004). Assumption (C) is stronger since we assume that $X_{i}$ is bounded below. Assumption (B) is stronger since Bonnisseau-Jamin (2004), only assume that $A_{t_{0}}^{C}$ is bounded for $t_{0}$ appearing in Assumption (BLS). Assumption (BLS) is also weaker than the original one since Part (BL) needs to hold true only on a bounded subset.

We now show that the economy $\mathcal{E}^{k}$ satisfies the assumptions of Theorem 3.2 for all $k \geq \hat{k}$. Assumption (PR) is a consequence of the continuity of the mappings $g_{j}^{k}, \Gamma^{k}, \sigma$ and the construction of $\gamma^{k}$.

Assumption (R') is a direct consequence of Lemma 3.6 (ii). Indeed $\left(y_{j}\right) \in A_{0}^{C}$ implies that $\theta^{C}\left(\left(\operatorname{proj}_{\mathbf{1}^{\perp}} y_{j}\right)\right) \leq 0$. Then, $\theta\left(\left(\operatorname{proj}_{\mathbf{1}^{\perp}} y_{j}\right)\right) \leq 0$ and $\left(\operatorname{proj}_{\mathbf{1}^{\perp}} y_{j}\right) \in\left(B_{\mathbf{1}^{\perp}}\left(0, r_{1}\right)\right)^{n}$. Consequently, $\varphi^{k}\left(p,\left(y_{j}\right)\right)=\left(g_{j}^{k}\left(\operatorname{proj}_{1^{\perp}} y_{j}\right)\right)$.

Let us now consider Assumption (BLS). Let $t_{0}>\max \left\{\theta^{C}(s) \mid s \in\right.$ $\left.\left(\bar{B}_{1^{\perp}}\left(0, r_{1}^{\prime}\right)\right)^{n}\right\}$. For Part (BS), let us consider $t \in\left[0, t_{0}\left[\right.\right.$ and $\left(p,\left(y_{j}\right)\right) \in$ $S^{C} \times \prod_{j=1}^{n} \partial Y_{j}$ such that $\left(y_{j}\right) \in A_{t}^{C}$ and $(p, \ldots, p) \in \varphi^{k}\left(p,\left(y_{j}\right)\right)$. We remark that the definition of $\varphi^{k}$ implies that $(p, \ldots, p) \in \varphi^{k}\left(p,\left(y_{j}\right)\right)$ is possible only if $s=\left(s_{j}\right)=\left(\operatorname{proj}_{1^{\perp}} y_{j}\right) \in \mathcal{M}$ since $\Gamma^{k}(s, t) \neq 0$ for all $(s, t) \in \partial M \times[0,1]$.

If $s \in \operatorname{int} \mathcal{M}=\left(B_{1^{\perp}}\left(0, r_{1}\right)\right)^{n}$, then $p=g_{j}^{k}\left(s_{j}\right)$ for all $j$. If $\sum_{j=1}^{n} y_{j}+$ $t \mathbf{1} \in \operatorname{int} X^{C}$, one directly gets that $p \cdot\left(\sum_{j=1}^{n} y_{j}+t \mathbf{1}\right)>\inf p \cdot X^{C}$. If $\sum_{j=1}^{n} y_{j}+t \mathbf{1} \in \partial X^{C}$, then $t=\Theta^{C}(s)$ and Lemma 3.6 (i) implies $p \notin$ $-N_{X^{C}}\left(-\Lambda_{0}^{C}\left(-\sum_{j=1}^{n} s_{j}\right)\right)$. Consequently, since $-\Lambda_{0}^{C}\left(-\sum_{j=1}^{n} s_{j}\right)=\sum_{j=1}^{n} y_{j}+$ $t \mathbf{1}, p \cdot\left(\sum_{j=1}^{n} y_{j}+t \mathbf{1}\right)>\inf p \cdot X^{C}$. 
If $s \in \partial \mathcal{M}$, from the definition of $\varphi^{k}$, there exists $\alpha \in[0,1]$ such that $p=\alpha g_{j}^{k}\left(s_{j}\right)+(1-\alpha)\left(p+\Gamma_{j}^{k}(s, 0)\right)$ for all $j$. If $\alpha=1$, the previous argument holds true again, and, we can conclude that $p \cdot\left(\sum_{j=1}^{n} y_{j}+t \mathbf{1}\right)>\inf p \cdot X^{C}$. If $\alpha=0$, one gets a contradiction with $\Gamma^{k}(s, 0) \neq 0$. Let us now consider the case where $\alpha \in] 0,1\left[\right.$. Then, $\alpha\left(p-g_{j}^{k}\left(s_{j}\right)\right)=(1-\alpha) \Gamma_{j}^{k}(s, 0)$ for all $j$. If $p \in-N_{X^{C}}\left(-\Lambda_{0}^{C}\left(-\sum_{j=1}^{n} s_{j}\right)\right), \delta=-\left(p-g_{j}^{k}\left(s_{j}\right)\right) \in \Delta^{k}(s)$, and, one gets a contradiction with Assertion (I) of Lemma 3.8 since $\delta \cdot \Gamma_{j}^{k}(s, 0)=$ $-\frac{1-\alpha}{\alpha} \sum_{j=1}^{n}\left\|\Gamma_{j}^{k}(s, 0)\right\|^{2}<0$. Hence $p \notin-N_{X^{C}}\left(-\Lambda_{0}^{C}\left(-\sum_{j=1}^{n} s_{j}\right)\right)$ and we end the proof as above to show that $p \cdot\left(\sum_{j=1}^{n} y_{j}+t \mathbf{1}\right)>\inf p \cdot X^{C}$.

We now consider Part (BL) of Assumption (BLS). Let $t \geq t_{0},\left(p,\left(y_{j}\right)\right) \in$ $S^{C} \times \prod_{j=1}^{n} \partial Y_{j}$, and, $\left(q_{j}\right) \in \varphi^{k}\left(p,\left(y_{j}\right)\right)$ such that $p \in-N_{X^{C}}\left(\sum_{j=1}^{n} y_{j}+t \mathbf{1}\right)$. Since $p \neq 0, \sum_{j=1}^{n} y_{j}+t \mathbf{1} \in \partial X^{C}$, which means that $\theta^{C}(s)=t$ with $s=$

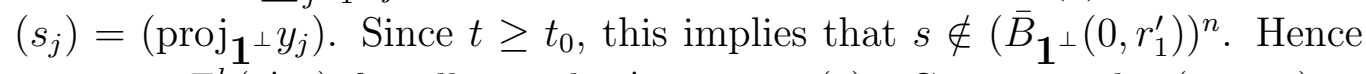
$q_{j}=p+\Gamma_{j}^{k}\left(s^{\prime}, 1\right)$ for all $j$ with $s^{\prime}=\operatorname{proj}_{\mathcal{M}}(s)$. Consequently, $\left(q_{j}-p\right)=$ $\Gamma^{k}\left(s^{\prime}, 1\right) \in-\operatorname{int} T_{\mathcal{M}}\left(s^{\prime}\right)$ from Assertion (II) of Lemma 3.8. For $\alpha>0$ close enough to $0, \hat{s}=s^{\prime}-\alpha \Gamma^{k}\left(s^{\prime}, 1\right) \in \operatorname{int} \mathcal{M}$. Let $\left(\hat{y}_{j}\right)=\Lambda(\hat{s})$. Since $\hat{s} \in \mathcal{M}$, $\theta^{C}(\hat{s}) \leq t_{0}$, hence $\left(\hat{y}_{j}\right) \in A_{t_{0}}^{C}$. Since $\Gamma^{k}\left(s^{\prime}, 1\right) \in\left(\mathbf{1}^{\perp}\right)^{n}$, one obtains:

$$
\begin{aligned}
\sum_{j=1}^{n}\left(q_{j}-p\right) \cdot\left(y_{j}-\hat{y}_{j}\right) & =\sum_{j=1}^{n} \Gamma_{j}^{k}\left(s^{\prime}, 1\right) \cdot\left(s_{j}-\hat{s}_{j}\right) \\
& =\sum_{j=1}^{n} \Gamma_{j}^{k}\left(s^{\prime}, 1\right) \cdot\left(s_{j}-s_{j}^{\prime}+\alpha \Gamma_{j}^{k}\left(s^{\prime}, 1\right)\right. \\
& \left.=\Gamma^{k}\left(s^{\prime}, 1\right) \cdot\left(s-s^{\prime}\right)+\alpha \| \Gamma_{(}^{k} s^{\prime}, 1\right) \|^{2}
\end{aligned}
$$

Since $s^{\prime}=\operatorname{proj}_{\mathcal{M}}(s), s-s^{\prime} \in N_{\mathcal{M}}\left(x^{\prime}\right)$. Since $\Gamma^{k}\left(s^{\prime}, 1\right) \in-\operatorname{int} T_{\mathcal{M}}\left(s^{\prime}\right)$, one gets $\Gamma^{k}\left(s^{\prime}, 1\right) \cdot\left(s-s^{\prime}\right) \geq 0$. Hence, since $\alpha\left\|\Gamma^{k}\left(s^{\prime}, 1\right)\right\|^{2}>0$, one finally obtains $\sum_{j=1}^{n}\left(q_{j}-p\right) \cdot\left(y_{j}-\hat{y}_{j}\right)>0$. This ends the proof that $\mathcal{E}^{k}$ satisfies the assumptions of Theorem 3.2.

Then, one deduces that for all $k \geq \hat{k}$, there exists a general pricing rule equilibrium $\left(\left(x_{i}^{k}\right),\left(y_{j}^{k}\right), p^{k}\right)$ in $\prod_{i=1}^{m} X_{i} \times \prod_{j=1}^{n} \partial Y_{j} \times S^{C}$ such that

(a) for every $i, x_{i}^{k}$ is a greater element for $\preceq_{i}$ in $B_{i}\left(p^{k},\left(y_{j}^{k}\right)\right)$;

(b) $\left(p^{k}, \ldots, p^{k}\right) \in \varphi^{k}\left(p^{k},\left(y_{j}^{k}\right)\right)$;

(c) $\sum_{i=1}^{m} x_{i}^{k}=\sum_{j=1}^{n} y_{j}^{k}+\omega$.

From Condition (c), one deduces that $\left(y_{j}^{k}\right)$ remains in the compact set $A_{0}$. Hence, $\left(\sum_{i=1}^{m} x_{i}^{k}\right)$ is bounded, which implies that the sequence $\left(x_{i}^{k}\right)$ is also bounded since the sets $X_{i}$ are bounded below. Consequently, the sequence $\left(\left(x_{i}^{k}\right),\left(y_{j}^{k}\right), p^{k}\right)$ is bounded. We assume without any loss of generality that this sequence converges to $\left(\left(x_{i}^{*}\right),\left(y_{j}^{*}\right), p^{*}\right) \in \prod_{i=1}^{m} X_{i} \times \prod_{j=1}^{n} \partial Y_{j} \times S^{C}$. 
Condition (c) implies that $\sum_{i=1}^{m} x_{i}^{*}=\sum_{j=1}^{n} y_{j}^{*}+\omega$ and for all $k \geq \hat{k}$, $\left(s_{j}^{k}\right)=\left(\operatorname{proj}_{1^{\perp}} y_{j}^{k}\right) \in M_{0} \subset\left[B_{1^{\perp}}\left(0, r_{1}\right)\right]^{n}=\operatorname{int} \mathcal{M}$. From the definition of $\varphi^{k}$ and $\gamma^{k}, \varphi^{k}\left(\left(p^{k},\left(y_{j}^{k}\right)\right)=\left(g_{j}^{k}\left(\Lambda_{j}^{k}\left(s_{j}^{k}\right)\right)\right)\right.$. From the definition of $g_{j}^{k}, g_{j}^{k}\left(\Lambda_{j}^{k}\left(s_{j}^{k}\right)\right) \in$ $N_{Y_{j}^{k}}\left(\Lambda_{j}^{k}\left(s_{j}^{k}\right)\right)$. From Lemma 3.4 (iii), since $\Lambda_{j}^{k}\left(s_{j}^{k}\right) \gg-\tau \mathbf{1}, N_{Y_{j}^{k}}\left(\Lambda_{j}^{k}\left(s_{j}^{k}\right)\right)=$ $N_{Z_{j}^{k}}\left(\Lambda_{j}^{k}\left(s_{j}^{k}\right)\right)$. From Lemma 3.5 , the sequence $\left(\Lambda_{j}^{k}\left(s_{j}^{k}\right)\right)$ converges to $s_{j}^{*}-$ $\lambda_{j}\left(s_{j}^{*}\right) \mathbf{1}=y_{j}^{*}$. From Assertion (iii) of Theorem 3.1 applied to $Z_{j}, p^{*}=$ $\lim g_{j}^{k}\left(\Lambda_{j}^{k}\left(s_{j}^{k}\right)\right) \in N_{Z_{j}}^{I}\left(y_{j}^{*}\right)$. From Lemma 3.2, $N_{Z_{j}}^{I}\left(y_{j}^{*}\right)=N_{Y_{j}}^{I}\left(y_{j}^{*}\right)$, hence $p^{*} \in$ $N_{Y_{j}}^{I}\left(y_{j}^{*}\right)$ for all $j$.

From above, one deduces that $r_{i}\left(p^{k},\left(y_{j}^{k}\right)\right)$ converges to $r_{i}\left(p^{*},\left(y_{j}^{*}\right)\right)$ and $r_{i}\left(p^{*},\left(y_{j}^{*}\right)\right)>\inf p^{*} \cdot X_{i}$ from Assumption $(\mathrm{R})$. Thus, one deduces that $x_{i}^{*}$ is a greater element for $\preceq_{i}$ in the budget set $B_{i}\left(p^{*},\left(y_{j}^{*}\right)\right)$ since $x_{i}^{k}$ is a greater element for $\preceq_{i}$ in the budget set $B_{i}\left(p^{k},\left(y_{j}^{k}\right)\right)$. This means that $\left(\left(x_{i}^{*}\right),\left(y_{j}^{*}\right), p^{*}\right)$ is a marginal pricing equilibrium of the economy $\mathcal{E}$.

\section{Appendix}

Proof of Lemma 3.2 The definition of $Z_{j}$ implies that it is a closed subset. It is bounded since $-\bar{\tau} \mathbf{1}$ is a lower bound and it is below $\Lambda_{j}\left(\bar{B}_{\mathbf{1}^{\perp}}\left(0, r_{2}\right)\right)$, which is a compact subset. Let $\tau>\underline{\tau}$ such that for all $s \in\left[\bar{B}_{1^{\perp}}\left(0, r_{2}\right)\right]^{n}$, for all $j=1, \ldots, n, \Lambda_{j}\left(s_{j}\right) \gg-\tau \mathbf{1}$. One easily checks that for all $z_{j} \in \partial Z_{j}$, $-\tau \mathbf{1}-z_{j}$ belongs to the interior of the Clarke's normal cone to $Z_{j}$ at $z_{j}$. So $Z_{j}$ is epi-lipschitzian.

For all $s_{j} \in \bar{B}_{1^{\perp}}\left(0, r_{2}\right)$, there exists $\rho>0$ small enough, such that $\operatorname{proj}_{\mathbf{1}^{\perp}}\left(B\left(\Lambda_{j}\left(s_{j}\right), \rho\right) \cap Y_{j}\right) \subset B_{\mathbf{1}^{\perp}}\left(0, r_{2}^{\prime}\right)$ and $B\left(\Lambda_{j}\left(s_{j}\right), \rho\right) \gg-\underline{\tau} \mathbf{1}$. For all $y_{j} \in B\left(\Lambda_{j}\left(s_{j}\right), \rho\right) \cap Y_{j}$, there exists $\alpha \geq 0$ such that $y_{j}=\Lambda_{j}\left(\operatorname{proj}_{\mathbf{1}^{\perp}}\left(y_{j}\right)\right)-\alpha \mathbf{1}$, and, $y_{j} \gg-\underline{\tau} \mathbf{1}$. Since $\Lambda_{j}\left(\operatorname{proj}_{\mathbf{1}^{\perp}}\left(y_{j}\right)\right) \in Z_{j}$, one concludes that $y_{j} \in Z_{j}$. Hence $B\left(\Lambda_{j}\left(s_{j}\right), \rho\right) \cap Y_{j} \subset B\left(\Lambda_{j}\left(s_{j}\right), \rho\right) \cap Z_{j}$. The converse inclusion is obvious since $Z_{j} \subset Y_{j}$. Consequently, $B\left(\Lambda_{j}\left(s_{j}\right), \rho\right) \cap Y_{j}=B\left(\Lambda_{j}\left(s_{j}\right), \rho\right) \cap Z_{j}$, which implies that $\Lambda_{j}\left(s_{j}\right) \in \partial Z_{j}$ and $N_{Z_{j}}^{I}\left(\Lambda_{j}\left(s_{j}\right)\right)=N_{Y_{j}}^{I}\left(\Lambda_{j}\left(s_{j}\right)\right)$.

Proof of Lemma 3.3 For $\rho>1$, we define the cone $C^{\rho}$ as follows:

$$
C^{\rho}=\left\{c \in \mathbb{R}^{\ell} \mid \rho \min _{h}\left\{\left(\operatorname{proj}_{\mathbf{1}^{\perp}} c\right)_{h}\right\}+\frac{c \cdot \mathbf{1}}{\ell} \geq 0\right\}
$$

One easily checks that $C^{\rho}$ is a closed convex cone included in $\{0\} \cup \mathbb{R}_{++}^{\ell}$ and $\mathbf{1} \in \operatorname{int} C$. Now, it suffices to choose $\rho>1$ close enough to 1 in order to get the second property. 
Since the set $\sum_{i=1}^{m} X_{i}-\omega$ is bounded below and $-\Lambda_{0}\left(\bar{B}_{e^{\perp}}\left(0, n r_{2}\right)\right.$ is closed, the set $K=\left\{u \in \mathbb{R}_{+}^{\ell} \mid \exists x \in \sum_{i=1}^{m} X_{i}-\omega, x+u \in-\Lambda_{0}\left(\bar{B}_{e^{\perp}}\left(0, n r_{2}\right)\right\}\right.$ is bounded. Hence, there exists $\rho>1$ close enough to 1 such that for all $u \in K, u+\eta \mathbf{1} \in \operatorname{int} C^{\rho}$.

For all $s \in \bar{B}_{\mathbf{1}^{\perp}}\left(0, n r_{2}\right),-s+\lambda_{0}(s) \mathbf{1}=-\Lambda_{0}(s)=x-\omega+u$ with $u \in$ $K$ and $x \in \sum_{i=1}^{m} X_{i}$. Consequently, $u+\eta \mathbf{1} \in \operatorname{int} C^{\rho}$, which implies that $-s+\left(\lambda_{0}(s)+\eta\right) \mathbf{1} \in \operatorname{int} X^{C}$. Hence $\lambda_{0}^{C}(s)<\lambda_{0}(s)+\eta$.

Proof of Lemma 3.4 The existence of $\underline{k}$ is a consequence of the compacity of $Z_{j}$, the fact that $N_{Z_{j}}^{I}\left(z_{j}\right) \subset \mathbb{R}_{+}^{\ell}$ for all $z_{j} \in Z_{j} \cap\left(\{-\tau \mathbf{1}\}+\mathbb{R}_{+}^{\ell}\right)$ and Property (iii) of the approximation. Indeed, by considering the intersection with the unit spere $S(0,1)$, we remark that $\mathbb{R}_{+}^{\ell} \cap S(0,1) \subset-\operatorname{int} C^{\circ}$.

Point (ii) is a direct consequence of the definition of $Y_{j}^{k}$ and the compactness of $Z_{j}^{k}$ for all $j$. The first assertion of Point (iii) is a direct consequence of the definition of $Y_{j}^{k}$ and of the fact that $Z_{j}^{k} \subset Y_{j}+\left\{\varepsilon_{j}^{k} 1\right\}$.

Now, let us consider an element $y_{j} \in Y_{j}^{k} \cap\left[\{-\tau \mathbf{1}\}+\mathbb{R}_{++}^{\ell}\right]$. If $y_{j} \notin Z_{j}^{k}$, there exists $c \in C \backslash\{0\}$ and $z_{j} \in Z_{j}^{k}$ such that $y_{j}=z_{j}-c$. Clearly, from the choice of $c, c \in \mathbb{R}_{++}^{\ell}$. Consequently, since $Z_{j}$ is compact, there exists $\zeta_{j} \in \partial Z_{j}^{k} \cap\left\{y_{j}+t c \mid t \geq 0\right\}$. From Point (i), $-c \in \operatorname{int} T_{Z_{j}^{k}}^{C}\left(\zeta_{j}\right)$. Hence, for $t>0$ small enough, $\zeta_{j}-t c \in Z_{j}^{k}$. From this remark and since $y_{j} \notin Z_{j}^{k}$, $\tilde{t}=\inf \left\{t \in \mathbb{R}_{+} \mid \zeta_{j}-t c \notin Z_{j}\right\}>0$ and $\zeta_{j}-\tilde{t} c \in \partial Z_{j}^{k}$. Since $-\tau \mathbf{1} \ll y_{j} \ll$ $\zeta_{j}-\tilde{t} c,-c \in \operatorname{int} T_{Z_{j}^{k}}\left(\zeta_{j}-\tilde{t} c\right)$. Consequently, for $t>\tilde{t}, t$ close enough to $\tilde{t}$, $\zeta_{j}-t c \in Z_{j}^{k}$. This contradicts the fact that $\tilde{t}=\inf \left\{t \in \mathbb{R}_{+} \mid \zeta_{j}-t c \notin Z_{j}\right\}$. Hence $y_{j} \in Z_{j}^{k}$.

Proof of Lemma 3.5 (i) $\lambda_{j}(s)-\varepsilon_{j}^{k} \leq \lambda_{j}^{k}(s)$ is a direct consequence of Lemma 3.4 (iii). For all $s \in \bar{B}_{\mathbf{1}^{\perp}}\left(0, r_{2}\right), s-\lambda_{j}(s) \mathbf{1} \in Z_{j} \subset Z_{j}^{k} \subset Y_{j}^{k}$, which implies the inequality $\lambda_{j}^{k}(s) \leq \lambda_{j}(s)$.

We now choose $\tilde{k} \geq \underline{k}$ in such a way that $M_{\varepsilon^{k}} \subset\left(B_{1^{\perp}}\left(0, r_{1}\right)\right)^{n}$ and $M_{\bar{t}+\eta+\varepsilon^{k}} \subset\left(B_{1^{\perp}}\left(0, r_{2}\right)\right)^{n}$. Such a choice is feasible since the set $M_{t}$ are compact, $t \rightarrow M_{t}$ is upper semi-continuous, $r_{1}$ and $r_{2}$ are well chosen and $\left(\varepsilon^{k}\right)$ converges to 0 .

Now, let us prove assertion (ii). If $\Theta^{k}(s) \leq 0$, then the first inequality above and the fact that $\lambda_{0} \leq \lambda_{0}^{C}$ imply that $\theta(s) \leq \varepsilon^{k}$ hence $M_{0}^{k} \subset M_{\varepsilon^{k}}$. The second inclusion comes from the choice of $\tilde{k}$.

For Assertion (iii), if $s \in\left(\bar{B}_{\mathbf{1}^{\perp}}\left(0, r_{1}\right)\right)^{n}$, then $\Theta^{k}(s)=\sum_{j=1}^{n} \lambda_{j}^{k}\left(s_{j}\right)+$ $\lambda_{0}^{C}\left(-\sum_{j=1}^{n} s_{j}\right)$, and from the second inequality in (i) and Lemma 3.3, one 
gets $\Theta^{k}(s) \leq \Theta(s)+\eta<\bar{t}+\eta$ since $-\sum_{j=1}^{n} s_{j} \in \bar{B}_{1^{\perp}}\left(0, n r_{1}\right)$.

The proof of Assertion (iv) is the same as the one of Assertion (ii).

Proof of Lemma 3.6 We prove that there exists $\hat{k}_{1} \geq \tilde{k}$ such that Assertion (i) holds true for all $k \geq \hat{k}_{1}$ and, then, that there exists $\hat{k}_{2} \geq \tilde{k}$ such that Assertion (ii) holds true for all $k \geq \hat{k}_{2}$. Hence, it suffices to take $\hat{k}$ greater than $\hat{k}_{1}$ and $\hat{k}_{2}$ to get the result.

Let us assume that $\hat{k}_{1}$ does not exist. Then, there exists a strictly increasing mapping $\psi$ from $\mathbb{N}$ to itself such that the sequence $\left(p^{\psi(k)}, s^{\psi(k)}\right) \in H \times$ $\left(\bar{B}_{\mathbf{1}^{\perp}}\left(0, r_{2}\right)\right)^{n}$ such that $\theta^{C}\left(s^{\psi(k)}\right) \geq 0$ and $p^{\psi(k)} \in-N_{X^{C}}\left(-\Lambda_{0}^{C}\left(-\sum_{j=1}^{n} s_{j}^{\psi(k)}\right)\right)$ and $p^{\psi(k)}=g_{j}^{\psi(k)}\left(s_{j}^{\psi(k)}\right)$ for all $j$. Since the normal cone of $X^{C}$ is included in $C^{\circ}$ and $\mathbf{1} \in \operatorname{int} C$, the sequence $\left(p^{\psi(k)}\right)$ remains in a compact subset of $H$. Consequently, without any loss of generality, we can assume that the sequence $\left(p^{\psi(k)}, s^{\psi(k)}\right)$ converges to $(\bar{p}, \bar{s}) \in H \times\left(\bar{B}_{\mathbf{1}^{\perp}}\left(0, r_{2}\right)\right)^{n}$. Let $\bar{y}_{j}=\Lambda_{j}\left(\bar{s}_{j}\right)$.

Note that $g_{j}^{\psi(k)}\left(s_{j}^{\psi(k)}\right) \in N_{Y_{j}^{\psi(k)}}^{C}\left(\Lambda_{j}^{\psi(k)}\left(s_{j}^{\psi(k)}\right)\right)$, and $s_{j}^{\psi(k)} \in \bar{B}_{\mathbf{1}^{\perp}}\left(0, r_{2}\right)$. Thus, $\Lambda_{j}^{\psi(k)}\left(s_{j}^{\psi(k)}\right) \geq \Lambda_{j}\left(s_{j}^{\psi(k)}\right) \gg-\tau \mathbf{1}$. From Lemma 3.4 (iii),

$$
N_{Y_{j}^{\psi(k)}}\left(\Lambda_{j}^{\psi(k)}\left(s_{j}^{\psi(k)}\right)\right)=N_{Z_{j}^{\psi(k)}}\left(\Lambda_{j}^{\psi(k)}\left(s_{j}^{\psi(k)}\right)\right)
$$

From Lemma 3.5, the sequence $\left(\Lambda_{j}^{\psi(k)}\left(s_{j}^{\psi(k)}\right)\right)$ converges to $\bar{s}_{j}-\lambda_{j}\left(\bar{s}_{j}\right) \mathbf{1}=\bar{y}_{j}$. From Theorem 3.1 (iii)applied to $Z_{j}, \bar{p}=\lim g_{j}^{\psi(k)}\left(\Lambda_{j}^{\psi(k)}\left(s_{j}^{\psi(k)}\right)\right) \in N_{Z_{j}}^{I}\left(\bar{y}_{j}\right)$. From Lemma 3.2, $N_{Z_{j}}^{I}\left(\bar{y}_{j}\right)=N_{Y_{j}}^{I}\left(\bar{y}_{j}\right)$, hence $\bar{p} \in N_{Y_{j}}^{I}\left(\bar{y}_{j}\right)$ for all $j$. From Assumption $(\mathrm{P}), N_{Y_{j}}^{I}\left(\bar{y}_{j}\right) \subset \mathbb{R}_{+}^{\ell}$, hence $\bar{p} \in S$.

From the closedness of the correspondence $-N_{X^{C}}$, one also deduces that $\bar{p} \in-N_{X^{C}}\left(-\Lambda_{0}^{C}\left(-\sum_{j=1}^{n} \bar{s}_{j}\right)\right)$ and from the continuity of $\theta^{C}, \theta^{C}(\bar{s}) \geq 0$. Consequently, there exists $x \in \sum_{i=1}^{m} X_{i}$ and $c \in C$ such that $\sum_{j=1}^{n} \bar{y}_{j}+$ $\theta^{C}(\bar{s}) \mathbf{1}=x-\omega+c$. Furthermore, for all $\xi \in X^{C}, \bar{p} \cdot(x-\omega+c) \leq \bar{p} \cdot \xi$. If $c \neq 0, c \in \mathbb{R}_{++}^{\ell}$ and $\bar{p} \cdot c>0$. Consequently $\bar{p} \cdot(x-\omega)<\bar{p} \cdot(x-\omega+c)$, which is impossible since $x-\omega \in X^{C}$. Hence, $\sum_{j=1}^{n} \bar{y}_{j}+\theta^{C}(\bar{s}) \mathbf{1}=x-\omega \in X$. This implies that $\theta^{C}(\bar{x}) \geq \theta(\bar{s})$. Furthermore, for all $\xi \in X$, there exists $x^{\prime} \in \sum_{i=1}^{m} X_{i}$ and $u \in \mathbb{R}_{+}^{\ell}$ such that $\xi=x^{\prime}-\omega+u$. Since $\bar{p} \cdot u \geq 0$ and $x^{\prime}-\omega \in X^{C}$, one gets $\bar{p} \cdot(x-\omega) \leq \bar{p} \cdot\left(x^{\prime}-\omega+u\right)=\bar{p} \cdot \xi$. Hence $\bar{p} \cdot\left(\sum_{j=1}^{n} \bar{y}_{j}+\theta^{C}(\bar{s}) \mathbf{1}\right)=\inf \bar{p} \cdot X$. This implies $\bar{p} \cdot \sum_{j=1}^{n} \bar{y}_{j}=\inf \bar{p} \cdot X-\theta^{C}(\bar{s}) \leq$ $\inf \bar{p} \cdot X-\theta(\bar{s})$, which contradicts Assumption (S) since $\theta(\bar{s})=d_{X}^{\infty}\left(\sum_{j=1}^{n} y_{j}\right)$.

We now come to the second part of the Lemma. If $\hat{k}_{2}$ does not exist, then, there exists a strictly increasing mapping $\psi$ from $\mathbb{N}$ to itself such that the 
sequence $\left(p^{\psi(k)}, s^{\psi(k)}, i^{\psi(k)}\right) \in H \times\left(\bar{B}_{1^{\perp}}\left(0, r_{2}\right)\right)^{n} \times\{1, \ldots, m\}$ such that, for all $k, \theta^{C}\left(s^{\psi(k)}\right) \leq 0, p^{\psi(k)}=g_{j}^{\psi(k)}\left(s_{j}^{\psi(k)}\right)$ for all $j$, and, $r_{i \psi(k)}\left(p^{\psi(k)},\left(\Lambda_{j}\left(s_{j}^{\psi(k)}\right)\right)\right) \leq$ $\inf p^{\psi(k)} \cdot X_{i \psi(k)}$. Using the same arguments as above, without any loss of generality, we can also assume that the sequence $\left(p^{\psi(k)}, s^{\psi(k)}\right)$ converges to $(\bar{p}, \bar{s})$ and that $i^{\psi(k)}$ is constant equal to $i$. We also deduces that $\bar{p} \in N_{Y_{j}}^{I}\left(\bar{y}_{j}\right)$ for all $j$ with $\bar{y}_{j}=\Lambda_{j}\left(\bar{s}_{j}\right)$, and $\theta^{C}(\bar{s}) \leq 0$. Since $X^{C} \subset X$, one has $\left(\bar{y}_{j}\right) \in A_{0}$ and $\left(\bar{p},\left(\bar{y}_{j}\right)\right) \in P E$. Hence, from Assumption $(\mathrm{R}), r_{i}\left(\bar{p},\left(\bar{y}_{j}\right)\right)>\inf \bar{p} \cdot X_{i}$. Consequently, there exists $\underline{x}_{i} \in X_{i}$ such that $r_{i}\left(\bar{p},\left(\bar{y}_{j}\right)\right)>\bar{p} \cdot \underline{x}_{i}$. The continuity of $r_{i}$ implies that $r_{i}\left(p^{\psi(k)},\left(\Lambda_{j}\left(s_{j}^{\psi(k)}\right)\right)\right)>p^{\psi(k)} \cdot \underline{x}_{i}$ for $k$ large enough. But this contradicts $r_{i}\left(p^{\psi(k)},\left(\Lambda_{j}\left(s_{j}^{\psi(k)}\right)\right)\right) \leq \inf p^{\psi(k)} \cdot X_{i}$ for all $k$.

Proof of Lemma 3.7 This is a direct consequence of the definition of $\Delta^{k}$, Lemma 3.6 and the fact that $\theta^{k}(s) \leq \theta^{C}(s)$ if $s \in\left(\bar{B}_{1^{\perp}}\left(0, r_{2}\right)\right)^{n}$ from Lemma 3.5 (i).

\section{References}

[1] Bonnisseau, J.M., (1992), "Existence of equilibria in presence of increasing returns: a synthesis", Journal of Mathematical Economics, 21, 441-452.

[2] Bonnisseau, J.M. and B. Cornet, (1990a), "Existence of Marginal Cost Pricing Equilibria : The Non-smooth Case", International Economic Review, 31, 685-708.

[3] Bonnisseau, J.M. and B. Cornet, (1990b), "Fixed-Point Theorems and Morse's Lemma for Lipschitzian Functions", Journal of Mathematical Analysis and Applications, 146, 318-332.

[4] Bonnisseau, J.M. and B. Cornet, (1990), "General equilibrium theory with increasing returns: the existence problem", in W.A. Barnett et al. (Eds.), Proceedings of the Sixth International Symposium on Economic Theory and Econometrics, Cambridge University Press, Cambridge, 65-82.

[5] Bonnisseau, J.M., B. Cornet and M.O. Czarnecki, (2005), "A tight definition for the marginal pricing rule", Cahier de la MSE, Université Paris 1. 
[6] Bonnisseau, J.M. and A. Jamin, (2004), "Equilibria with Increasing Returns : Sufficient Conditions on Bounded Production Allocations", Cahier de la MSE 2004-17, Université Paris 1.

[7] Clarke, F., 1983, Optimization and Nonsmooth Analysis". NewYork : John Wiley.

[8] Cornet, B., (1990), "Existence of equilibria in economies with increasing returns," Contributions to Operation Research and Economics: The Twentieth Anniversary of CORE, MIT Press, Cambridge, MA.

[9] Cornet, B. and M.O. Czarnecki (2001), "Existence of generalized equilibria," Nonlinear Analysis, 44, Ser. A : Theory Methods, $555-574$.

[10] G. Debreu (1959), Theory of Value, Yale University Press, New Haven.

[11] G. Debreu (1962), "New Concepts and Techniques for Equilibrium Analysis", International Economic Review, 3, 257-273.

[12] Guesnerie, R., (1975), "Pareto-optimality in nonconvex economies", Econometrica, 43, 1-29.

[13] Jouini, E., (1988), "A remark on Clarke's normal cone and the marginal cost pricing rule", Journal of Mathematical Economics , 17, 309-315.

[14] Jouini, E., (1990), "Functions with constant generalized gradients", Journal of Mathematical Analysis and Applications, 148, 121-130.

[15] Khan, M.A., (1999), "The Mordukhovich Normal Cone and the Foundations of Welfare Economics," Journal of Public Economic Theory, 1, 309-338. 